\title{
Quantum Threshold for Optomechanical Self-Structuring in a Bose-Einstein Condensate
}

\author{
G.R.M. Robb, E. Tesio, G.-L. Oppo, W.J. Firth, and T. Ackemann \\ SUPA and Department of Physics, University of Strathclyde, Glasgow G4 ONG, Scotland, UK \\ R. Bonifacio \\ INFN-LNF, Italy $\&$ Universidade Federal da Paraiba, Brazil
}

(Dated: March 13, 2015)

\begin{abstract}
Theoretical analysis of the optomechanics of degenerate bosonic atoms with a single feedback mirror shows that self-structuring occurs only above an input threshold which is quantum-mechanical in origin. This threshold also implies a lower limit to the size (period) of patterns which can be produced in a condensate for a given pump intensity. These thresholds are interpreted as due to quantum rigidity of Bose-Einstein condensates which has no classical counterpart. Above threshold, the condensate self-organizes into an ordered supersolid state with a spatial period self-selected by optical diffraction.
\end{abstract}

Self-organized patterns and structures which arise due to the simultaneous presence of optical nonlinearity and diffraction have been predicted and observed in a variety of media, including hot atomic vapours, for a number of years [1-5]. In the case of hot atomic vapours these phenomena rely solely on spatial modulation of the internal states of the atoms (electronic or Zeeman states) with their centre-of-mass motion playing no significant role. In cold atomic gases the additional presence of optical dipole forces leads to density self-organization and removes the requirement for an intrinsic optical nonlinearity. We will address the formation of a supersolid state in a quantum degenerate atomic gas from spontaneous symmetry breaking. The supersolid forms due to interaction mediated by the optomechanical forces of the light field and has a length scale determined by diffraction. Previously, optomechanical nonlinearity has been shown to give rise to collective atom-light interactions and instabilities which have been given a variety of names e.g. Collective Atomic Recoil Lasing (CARL) [6-8] and Superradiant Rayleigh Scattering (SRS) [9-11] but have similar physical characteristics to transversely pumped cavities, both with cold thermal and quantum degenerate matter [12-18]. The common features of these schemes are a pump beam which is scattered by the gas into an externally imposed mode (often selected by a cavity). The interference between this mode and the pump then provides a modulated light pattern, which via dipole forces leads to a spatial rearrangement of atoms. The emerging density gratings resulting from the interference of this mode and the pump then provide positive feedback by scattering photons into the self-sustained mode. In these arrangements, the spatial scale of the emerging structure is predetermined by the light wavelength and the geometrical configuration. From the photonics and quantum optics point of view, cold atomic systems enable the prospect of very low thresholds [19-22] offering the attractive prospect of all-optical control of symmetry breaking at the single photon level [19].
Alternative and naturally multimode schemes are possible in cold atoms, where spatial organization emerges in the plane transverse to the propagation of a single beam, with self-selected scales. It is in fact expected that atomic transport due to dipole forces can lead to nonlinear effects in cold atoms analogous to the Kerr effect in the hot-atoms case [23-25]. The study of transverse optomechanical instabilities in cold atoms has until recently been limited to the case where strong velocity damping is provided by optical molasses [20, 24, 25]. A similar optomechanical nonlinearity involving strong momentum damping is also present in soft matter systems e.g. suspensions of nanoparticles [26-29], but compared to soft matter systems cold atoms have the advantage that the dynamics can be studied without viscous damping of motion, allowing for a coherent, dissipation-free evolution. Recently [30], it was demonstrated that a system comprising a cold, thermal gas of $\mathrm{Rb}$ atoms and a single feedback mirror could produce spontaneous symmetry breaking resulting in hexagonal optical and atomic density patterns due to an instability arising from optical dipole forces in the absence of momentum damping. The theoretical analysis of this self-structuring process was described in [31] using a model where the cold atomic gas is described as a collisionless gas of classical particles.

Many recent studies of cold atom - light interactions have involved atomic gases which are cooled to sub-recoil temperatures, usually Bose-Einstein condensates (BEC) where a classical model of the atom-light interaction is insufficient and a quantum model, in which the de-localized atoms are treated as a matter wave, has to be adopted. It has been shown in studies of e.g. CARL [9, 32-34] that quantum effects can significantly change the nature of the atom-light interaction due to the discrete nature of the momentum exchange between the atoms and light. In addition, self-induced dipole forces which result in optical Kerr-like mutual self-focussing of co-propagating optical and matter wave beams were investigated theoreti- 
cally in [23] and CARL-induced self-focussing in a BEC was investigated theoretically in [35]. In this letter we demonstrate that quantum effects can significantly affect the process of optomechanical diffractive self-structuring observed in [30] and described classically in [31]. In what follows we present a quantum model of the pattern formation/self-structuring process, highlight the specifically quantum features which result and show that it can be described as a transition to a supersolid state with a self-selected spatial period.

The setup considered is shown schematically in fig. 1, where a BEC is illuminated by an optical beam which, after passing through the gas, is reflected by a single feedback mirror. The two far off-resonant, counterprop-

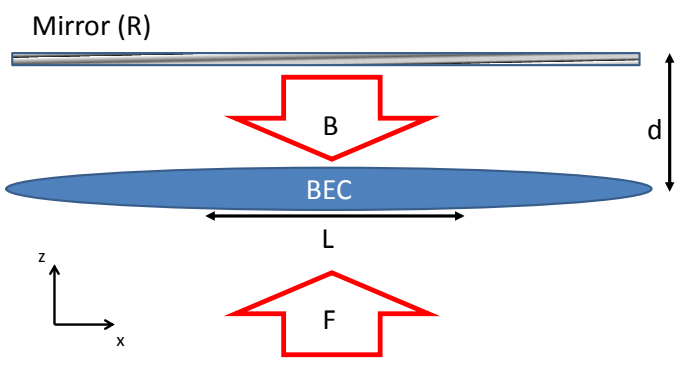

FIG. 1: Schematic diagram of the single mirror feedback scheme.

agating optical fields, represented by $(F, B)$, produce an optical potential which can be written as

$$
V(x)=\frac{\hbar \delta}{2} s(x, t)=\frac{\hbar \Gamma \Delta}{4} s(x, t)
$$

where $\delta=\omega-\omega_{a}$ is the field-atom detuning, $\omega, \omega_{a}$ are the pump laser and atomic resonance frequencies respectively, $\Delta=\frac{2 \delta}{\Gamma}, \Gamma$ is the atomic transition linewidth, $s=\left(|F|^{2}+|B|^{2}\right)$ and $|F, B|^{2}=\frac{1}{\Delta^{2}} \frac{I_{F, B}}{I_{\mathrm{sat}}}$ are the forward and backward field intensities scaled with respect to the saturation intensity at detuning $\delta$. It has been assumed that $\Delta \gg 1$ and that consequently $s \ll 1$ so that the atoms remain in their ground state. In addition, longitudinal grating effects due to interference between the counterpropagating optical fields on the transverse pattern formation process are neglected, as in [30, 31]. In previous studies of pattern formation in Kerr media, e.g. [36], the effect of including longitudinal grating effects on transverse pattern formation is simply to reduce minimum thresholds typically by approximately a factor of 2. Using eq. (1), the Schrodinger equation describing the evolution of the BEC wavefunction in this potential can be written as

$$
i \frac{\partial \psi(x, t)}{\partial t}=\left[-\frac{\hbar}{2 m} \frac{\partial^{2}}{\partial x^{2}}+\frac{\delta}{2} s(x, t)\right] \psi(x, t) .
$$

where for simplicity we have neglected atomic collisions, so that the atom-light coupling is the dominant source of nonlinearity, and assumed an untrapped BEC. Extensions of this model to cases where both atom-light and atom-atom coupling (collisions) are included, and where a trapping potential is included will be the subject of future extended studies. We consider a transverse domain of length $L$ so that $\psi(x, t), F, B$ are periodic with period $L$. In this case it is possible to write $\psi(x, t), F, B$ as

$$
\begin{aligned}
\psi(x, t) & =\sum_{n} c_{n}(t) e^{i n k_{L} x} \\
(F, B)(x, z, t) & =\sum_{n}\left(F_{n}(z, t), B_{n}(z, t)\right) e^{i n k_{L} x}
\end{aligned}
$$

where $k_{L}=\frac{2 \pi}{L}$ is the wavenumber associated with transverse period/domain length $L$ and $n$ is an integer. Substituting for $\psi(x, t), F(x, t), B(x, t)$ in eq. (2) using eq. (3) and eq. (4) results in

$$
i \frac{d c_{n}(t)}{d t}=\omega_{r} n^{2} c_{n}+\frac{\delta}{2} \sum_{m, p}\left(F_{m} F_{p}^{*}+B_{m} B_{p}^{*}\right) c_{n-m+p}
$$

where the intrinsically quantum parameter $\omega_{r}=\frac{\hbar k_{L}^{2}}{2 m}$ is the "recoil frequency" associated with wavenumber $k_{L}$.

The evolution of the forward-propagating optical field in the BEC is described by

$$
\frac{\partial F(x, z, t)}{\partial z}=-i \frac{b_{0}}{2 \Delta L_{z}}|\psi(x, t)|^{2} F(x, z, t)
$$

where $b_{0}$ is the optical thickness of the BEC cloud at resonance, $L_{z}$ is the longitudinal thickness of the cloud

$$
\int_{0}^{L}|\psi(x, t)|^{2} d x=L
$$

and it has been assumed that the atomic gas is sufficiently thin that diffraction within it can be neglected (thin medium approximation). Substituting for $\psi$ and $F$ using eq. (3) and (4) respectively results in

$$
\frac{\partial F_{n}(z, t)}{\partial z}=-i \frac{b_{0}}{2 \Delta L_{z}} \sum_{m, p} F_{m} c_{p} c_{p-n+m}^{*}
$$

As the field propagates a distance $2 d$ from the sample to the mirror and back, optical diffraction plays a crucial role by converting phase modulations to amplitude modulations and consequently optical dipole forces. The relation between the forward and backward fields when it leaves the BEC (where $z=L_{z}$ ) is

$$
B_{n}\left(z=L_{z}\right)=\sqrt{R} F_{n}\left(z=L_{z}\right) e^{-i n^{2} \Theta}
$$


where $\Theta=\frac{k_{L}^{2} d}{k_{0}}$ is the phase shift accumulated due to propagation from the BEC to the mirror and back when $n=1$.

The evolution of the system is therefore completely described by eq. (5), (7) and (8).

Assuming that all spatially varying quantities (i.e. all modes with index $\neq 0$ ) are small fluctuations and retaining only first-order terms in eq. (5) we obtain

$$
\begin{aligned}
& i \frac{d c_{n}(t)}{d t}=\omega_{r} n^{2} c_{n} \\
& +\frac{\delta}{2}\left(F_{0} F_{-n}^{*}+B_{0} B_{-n}^{*}+F_{0}^{*} F_{n}+B_{0}^{*} B_{n}\right) .
\end{aligned}
$$

The forward field evolution, described by eq. (7) can similarly be approximated by

$$
\frac{\partial F_{n}(z, t)}{\partial z}=-i \frac{b_{0}}{2 \Delta L_{z}}\left[F_{0}(z, t)\left(c_{0} c_{n}^{*}+c_{0}^{*} c_{n}\right)+F_{n}(z, t)\right]
$$

where $n \neq 0$ and the fact that $c_{0} c_{0}^{*}=1$ (from normalisation of $\psi$ ) has been used. The spatially-uniform component of the field, $F_{0}$, evolves according to

$$
\frac{\partial F_{0}(z, t)}{\partial z}=-i \frac{b_{0}}{2 \Delta L_{z}} F_{0}(z, t)
$$

which has the solution

$$
F_{0}(z, t)=F_{0}(z=0, t) e^{-i \frac{b_{0} z}{2 \Delta L_{z}}}
$$

so eq. (10) can be solved for $F_{n}(z)$, giving

$$
F_{n}\left(z=L_{z}\right)=-i \frac{b_{0}}{2 \Delta} F_{0}\left(L_{z}\right)\left(c_{0} c_{-n}^{*}+c_{0}^{*} c_{n}\right) .
$$

Substituting $F_{n}$ and $B_{n}$ into eq. (9) using eq. (11) and (8) respectively gives

$$
\frac{d c_{n}}{d t}=i \omega_{r} n^{2} c_{n}+i \frac{b_{0} p_{0} \Gamma}{4} \sin \left(n^{2} \Theta\right)\left(c_{0} c_{-n}^{*}+c_{0}^{*} c_{n}\right)
$$

where $p_{0}=\frac{1}{\Delta^{2}} \frac{I_{\mathrm{pump}}}{I_{\mathrm{sat}}}$ is the pump saturation parameter. Looking for solutions $\left(c_{0} c_{-n}^{*}+c_{0}^{*} c_{n}\right) \propto e^{\lambda t}$ results in the dispersion relation

$$
\lambda^{2}+\omega_{r}^{2} n^{4}-\frac{n^{2} \omega_{r} b_{0} p_{0} \Gamma R}{2} \sin \left(n^{2} \Theta\right)=0 .
$$

Note that in the continuous limit where $n k_{L} \equiv q$ is a continuous wavenumber, then the dispersion relation in eq. (13) can be written as

$$
\lambda^{2}+\omega_{r_{q}}^{2}-\frac{\omega_{r_{q}} b_{0} p_{0} \Gamma R}{2} \sin \left(q^{2} d / k_{0}\right)=0 .
$$

where $\omega_{r_{q}}=\frac{\hbar q^{2}}{2 m}$ is the recoil frequency associated with emission and absorption of photons with transverse momentum $\hbar q$. Consequently, an instability threshold condition $\operatorname{Re}(\lambda)=0$ can be written as a threshold in the pump saturation parameter

$$
p_{0} \equiv p_{\text {th }}=\frac{2 \omega_{r_{q}}}{b_{0} \Gamma R \sin \left(q^{2} d / k_{0}\right)}
$$

In contrast to the instability threshold demonstrated here, the model described in [31], which assumed a cold classical gas of atoms, predicts a threshold $\propto T$ at low temperature i.e. zero threshold at zero temperature. The existence of a finite minimum threshold in the BEC case is therefore quantum mechanical in origin. The threshold may be interpreted physically as due to the intrinsic velocity of the BEC wavepacket i.e. the kinetic part of the Schrodinger equation, which acts to spread the wavefunction and opposes the formation of any density modulation. This threshold can also be written as a threshold in wavenumber $q<q_{\mathrm{dom}}$ of $q_{\mathrm{dom}}$, where

$$
q_{d o m}=\sqrt{\frac{m b_{0} \Gamma R p_{0}}{\hbar}} .
$$

is the dominant wavenumber for which the instability growth rate is largest as $\sin \left(q_{d o m}^{2} d / k_{0}\right)=1$. Consequently there exists a lower limit to the spatial size/period of the periodic structure, $L_{\min } \approx 2 \pi / q_{d o m}$, which the BEC will sustain. The growth rate, $G(q)=$ $\operatorname{Re}(\lambda)$, is given by

$$
G(q)=R e(\lambda)=\sqrt{\frac{\omega_{r_{q}} b_{0} p_{0} \Gamma R}{2} \sin \left(q^{2} d / k_{0}\right)-\omega_{r_{q}}^{2}}
$$

which requires $\sin \left(q^{2} d / k_{0}\right)>0$ for $G(q)>0$. Note that far above threshold i.e. for pump intensities such that $p_{0}>>p_{\text {th }}$, the instability growth rate of eq. (17) reduces to

$$
G=R e(\lambda) \approx \sqrt{\frac{\omega_{r_{q}} b_{0} p_{0} \Gamma R}{2} \sin \left(q^{2} d / k_{0}\right)}
$$

where it has been assumed that $\sin \left(q^{2} d / k_{0}\right)>0$. It can be shown that this growth rate is identical to that of the classical growth rate derived in [31] at zero temperature.

Fig. 2 shows the dependence of pump threshold $\left(p_{\mathrm{th}}\right)$ on wavenumber $\left(\frac{q^{2} k_{0}}{d}\right)$ and, for a fixed pump intensity, the dependence of instability growth rate $(G)$ on wavenumber as calculated from eq. (17). It can be seen that there are multiple instability regions or balloons which result from the argument of the $\sin \left(q^{2} k_{0} / d\right)$ changing by multiples of $2 \pi$. In contrast to the prediction of the classical model [31], the instability balloons in the quantum case are not degenerate but have threshold minima which increase with $q^{2}$. The dependence of threshold intensity on wavenumber is very different from that of the classical model of [31], which predicts instability balloons with a degenerate minimum threshold which becomes zero at zero temperature. Note that the quantum model predicts a minimum threshold at $q=0$, but the growth rate here is zero. There is a finite band of unstable wavenumbers $q$ with $q>0$ with the maximum growth rate occurring at $q=q_{\text {dom }}$.

Figs. 3 and 4 show snapshots of the evolution of the field intensities/saturation parameter, $s$, and the BEC 

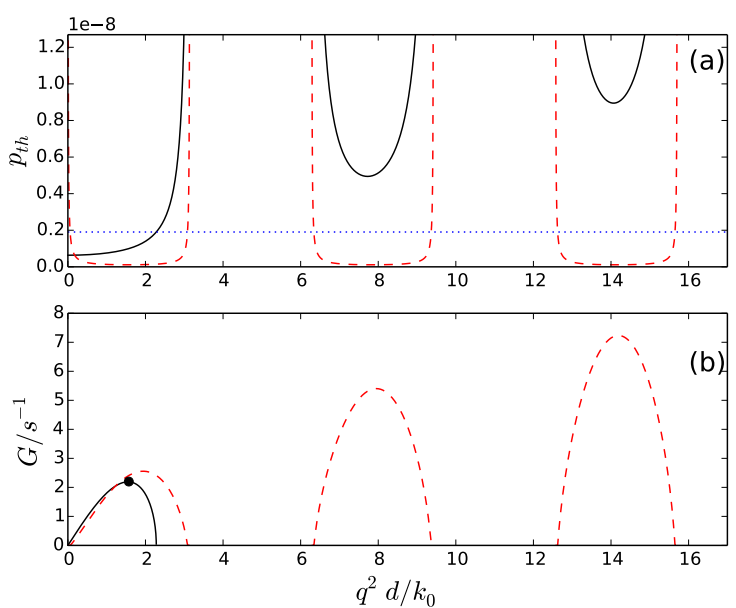

FIG. 2: (a) Threshold pump intensity saturation parameter $p_{\text {th }}$ as a function of $q^{2} k_{0} / d$ as calculated from eq. (15) (solid line) and from the classical model of [31], for a small but finite temperature $T=1 \times 10^{-12} \mathrm{~K}$ (dashed line). (b) Growth rate $G$ as a function of $q^{2} k_{0} / d$ for $p_{0}=1.9 \times 10^{-9}$ (dotted line in upper pane) as calculated from eq. (17) (solid line) and from the classical model of [31] for $T=1 \times 10^{-12} \mathrm{~K}$ (dashed line). Parameters used are $b_{0}=20,|\Delta|=100, d / k_{0}=2.48 \times$ $10^{-10} \mathrm{~m}^{2}, R=0.99, \Gamma=3.77 \times 10^{7} \mathrm{~s}^{-1}$.

density distribution, $|\Psi|^{2}$, for parameters such that the pump intensity exceeds the threshold in eq. (15), resulting in the simultaneous formation of a periodic modulation in light intensity and BEC density and initiated by noise. The dispersion relation eq.(13) predicts that for the parameters used, of all the modes included in the simulation those with $n \leq 6$ are above threshold. It can be seen that the modulation produced corresponds to $n=5$, which has maximum growth rate due to the value of $\Theta$ used, as indicated by the dot in fig. 2 (b). In fig. 3, the pump field is red-detuned with respect to the atomic resonance $(\Delta<0)$, which results in the BEC being attracted to regions of maximum light intensity. Conversely in fig. 4, the pump field is blue-detuned with respect to the atomic resonance $(\Delta>0)$, which results in the atoms being attracted to regions of minimum light intensity. Simulations of a cold classical gas would differ significantly from those shown in figs. 3 and 4 as they would show growth of larger-q patterns with shorter spatial periods. In both cases the pattern amplitude of the BEC grows exponentially and then fluctuates around a finite value, showing an asymptotic structured state.

In conclusion, we have demonstrated that when a zerotemperature BEC interacts with counterpropagating optical fields in the presence of a single feedback mirror, a self-structuring instability occurs which results in spontaneous transverse modulation of the optical intensity and the BEC. In contrast to the case of a classical gas, in the case of the quantum gas/ BEC there is an instability threshold at zero-temperature which suppresses
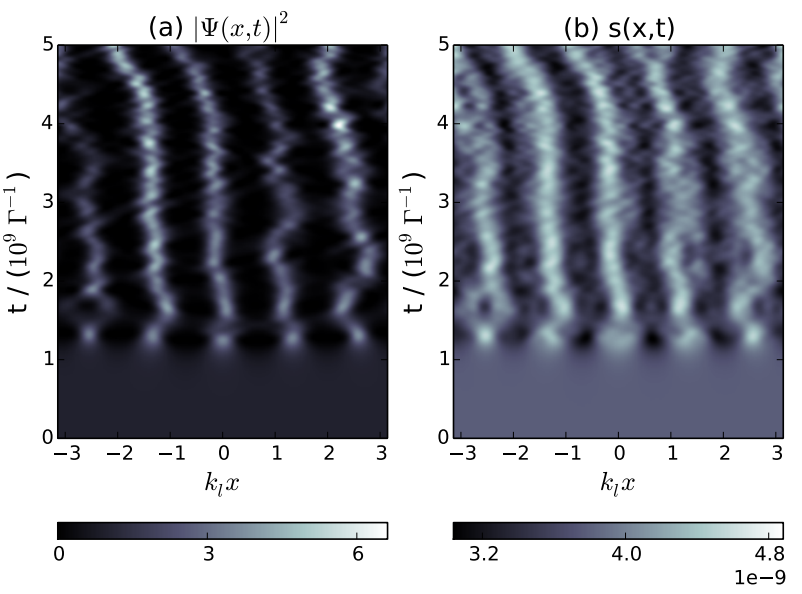

FIG. 3: Evolution of (a) the BEC density distribution, $|\Psi(x, t)|^{2}$, and (b) the optical intensity , $s(x, t)$, during the selfstructuring instability when the pump-field is red-detuned. Parameters used are $\Theta=\pi / 50, b_{0}=20, \Delta=-100, \Gamma / \omega_{r}=$ $2.53 \times 10^{9}, R=0.99$ and $p_{0}=1.9 \times 10^{-9}$.
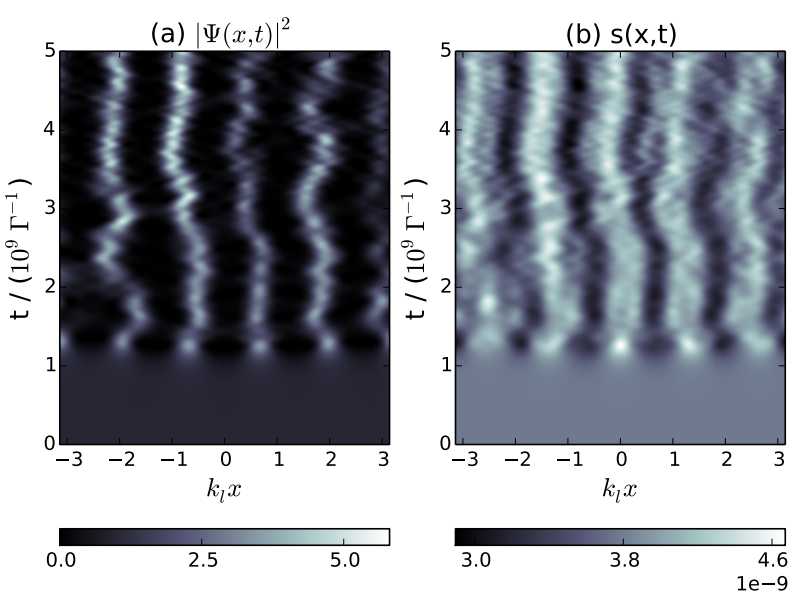

FIG. 4: Evolution of (a) the BEC density distribution, $|\Psi(x, t)|^{2}$, and (b) the optical intensity , $s(x, t)$, during the selfstructuring instability when the pump-field is blue-detuned. Parameters used are as for fig. 3 with the exception that $\Delta=100$.

high-q modes which are predicted to grow by the classical model. A consequence of this is that for a given pump field intensity, there is a lower limit to the transverse size of the structure or pattern which can be produced in a BEC. A physical explanation for this limit is that the dispersion of the BEC wavepacket stabilises the tendency of the initially uniform BEC density distribution to become modulated as a result of instability. A possible interpre- 
tation of this limit is as a 'healing length' of structures in the coupled BEC-light system analogous to the usual healing length associated with the scattering length of a collisional BEC. Just as the healing length of a BEC is an important length scale for structures such as solitons in BECs $[37,38]$ this optomechanical healing length should play an analogous role for optomechanically induced structures similar to those proposed in e.g. [39] but which involve coherent light and matter.

Another interesting aspect of the results presented here is the connection with generic phase transition phenomena in quantum gases. Several recent experiments involving BECs in optical cavities [16, 34, 40] have explored connections with Dicke Hamiltonian models and with associated phenomena e.g. transitions to a supersolid state [40]. It can be shown [41] that the BEC with single-mirror feedback scheme considered here can also be mapped onto an extended, multi-mode Dicke Hamiltonian model. Similarly, the transition to a spatially modulated state has characteristics of a transition to a supersolid in the sense of [40]. In common with [40] the transition occurs due to long-range optically-mediated forces but an important distinction is that the spatially ordered 'supersolid' state observed here has a spatial period with a length scale which is self-selected and not externally imposed.

The authors acknowledge useful discussions with Andrew Daley and financial support from the Leverhulme Trust (research grant F/00273/0) and the Engineering and Physical Sciences Research Council (for GRMR grant EP/H049339).

[1] M.C. Cross and P.C. Hohenberg, Rev. Mod. Phys. 65, 851 (1993)

[2] G. Grynberg, E. Le Bihan, P. Verkerk, P. Simoneau, J.R.R. Leite, D. Bloch, S. Le Boiteux and M. Ducloy, Opt. Commun. 67, 363 (1988)

[3] G.L. Lippi, T. Ackemann, L.M. Hoffer and W. Lange, Chaos, Soliton \& Fractals 4, 1419 (1994) and 1433 (1994)

[4] G. Giusfredi, J. F. Valley, R. Pon, G. Khitrova, H. M. Gibbs, J. Opt. Soc. Am. B 5, 1181 (1988)

[5] W.J. Firth, J. Mod. Opt. 37, 151 (1990)

[6] R. Bonifacio and L. De Salvo, Nucl. Instrum. and Meth. in Phys. Res. A 341, 360 (1994).

[7] C. von Cube, S. Slama, D. Kruse, C. Zimmermann, Ph.W. Courteille, G.R.M. Robb, N. Piovella, R. Bonifacio, Phys. Rev. Lett. 93, 083601 (2004)

[8] S. Slama, S. Bux, G. Krenz, C. Zimmermann and Ph.W. Courteille, Phys. Rev. Lett. 98, 053603 (2007)

[9] S. Inouye, A.P. Chikkatur, D.M. Stamper-Kurn, J. Stenger, D.E. Pritchard and W. Ketterle, Science 285, 571 (1999)

[10] D. Schneble, Y. Torii, M. Boyd, E.W. Streed, D.E. Pritchard and W. Ketterle, Science 300, 475 (2003)

[11] H. Kessler, J. Klinder, M. Wolke, and A. Hemmerich, Phys. Rev. Lett. 113, 070404 (2014)
[12] P. Domokos and H. Ritsch, Phys. Rev. Lett. 89, 253003 (2002)

[13] A. T. Black, H. W. Chan, and V. Vuletić, Phys. Rev. Lett. 91, 203001 (2003).

[14] S. Gupta, K. L. Moore, K. W. Murch, and D. M. Stamper-Kurn, Phys. Rev. Lett. 99, 213601 (2007) .

[15] F. Brennecke, S. Ritter, T. Donner \& T. Esslinger, Science 322, 235 (2008)

[16] K. Baumann, C. Guerlin, F. Brennecke and T. Esslinger, Nature 464, 1301 (2010).

[17] T. Botter, D.W.C. Brooks, S. Schreppler, N. Brahms, and D.M. Stamper-Kurn, Phys. Rev. Lett. 110, 153001 (2013)-.

[18] H. Ritsch, P. Domokos, F. Brenneke and T. Esslinger Rev. Mod. Phys. 85, 553 (2013).

[19] J.A. Greenberg, B.L. Schmittberger and D.J. Gauthier, Opt. Express 19, 22535-22549 (2011).

[20] E. Tesio, G. R. M. Robb, T. Ackemann, W. J. Firth, and G.-L. Oppo, Phys. Rev. A 86, 031801(R) (2012)

[21] H. Tercas, J. T. Mendona, and R. Kaiser, Eur. Phys. Lett. 8953001 (2010)

[22] J. T. Mendonca and R. Kaiser, Phys. Rev. Lett. 108, 033001 (2012).

[23] M. Saffman, Phys. Rev. Lett. 81, 65 (1998).

[24] G. A. Muradyan, Y. Wang, W. Williams and M. Saffman, Nonlinear guided waves topical meeting technical digest, paper ThB29 (2005)

[25] M. Saffman and Y. Wang, Lect. Notes Phys. 751, 361 (2008)

[26] P.W. Smith, A. Ashkin, and W.J. Tomlinson, Opt. Lett. 6, 284 (1981)

[27] A. Askhin, J.M. Dziedzic, and P.W. Smith, Opt. Lett. 7, $276(1982)$

[28] S.M. Wiggins, G.R.M. Robb, B.W.J. McNeil, D.R. Jones, D.A. Jaroszynski and S.J. Jamieson, J. Mod. Opt. 49, 997 (2002) Phys. Rev. E 67, 025603 (2003)

[29] P.J. Reece, E.M. Wright, and K. Dholakia, Phys. Rev. Lett. 98, 203902 (2007)

[30] G. Labeyrie , E. Tesio, P. M. Gomes, G-L. Oppo, W.J. Firth, G.R.M. Robb , A.S. Arnold, R. Kaiser \& T. Ackemann, Nat. Phot. 8321 (2014).

[31] E. Tesio, G.R.M. Robb, T. Ackemann, W.J. Firth and G.-L. Oppo, Phys. Rev. Lett. 112, 043901 (2014).

[32] N. Piovella, M. Gatelli and R. Bonifacio, Opt. Comm. 194, 167 (2001).

[33] L. Fallani, C. Fort, N. Piovella, M. M. Cola, F. S. Cataliotti, M. Inguscio and R. Bonifacio, Phys. Rev. A 71, $033612(2005)$.

[34] D. Schmidt, H. Tomczyk, S. Slama \& C. Zimmermann, Phys. Rev. Lett. 112, 115302 (2014).

[35] N. Piovella, L. Volpe, M. M. Cola \& R. Bonifacio, Laser Physics 17, 174 (2007)

[36] W. J. Firth, A. Fitzgerald and C. Pare, JOSA B 7, 1087 (1990).

[37] L. Khaykovich, F. Schreck, G. Ferrari, T. Bourdel, J. Cubizolles, L. D. Carr, Y. Castin, C. Salomon, Science 2961290 (2002).

[38] K.E. Strecker, G. B. Partridge, A. G. Truscott, \& R. G. Hulet, Nature 417150 (2002).

[39] E. Tesio, G.R.M. Robb, T. Ackemann, W.J. Firth \& G.L. Oppo, Opt. Expr. 21, 26144 (2013) .

[40] R. Mottl, F. Brennecke, K. Baumann, R. Landig, T. Donner \& T. Esslinger, Science 336, 1570 (2012).

[41] E. Tesio, PhD thesis, University of Strathclyde (2014). 